\title{
Real-World Experience of Immunotherapy from India in Recurrent Squamous Cell Carcinoma of Head and Neck Cancer
}

\author{
Waseem Abbas ${ }^{1}$ Saurabh Gupta ${ }^{1}$ Vineeta Goel ${ }^{1} \quad$ Ranga R. Rao $^{1}$ Promila Pankaj ${ }^{1}$ Devashish Tripathi \\ Pratik P. Patil ${ }^{1}$ Swati Popli ${ }^{1}$ \\ ${ }^{1}$ Department of Oncology, Max Super Speciality Hospital, Delhi, \\ India \\ Address for correspondence Waseem Abbas, DNB, Department of \\ Oncology, Max Super Speciality Hospital Shalimar Bagh. FC-50 C and \\ D Block, Shalimar Bagh 110008, Delhi, India \\ (e-mail:drabbasdoc@gmail.com).
}

South Asian J Cancer 2021;10:72-75.

\section{Abstract}

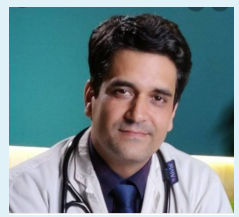

Waseem Abbas
Keywords

- immunotherapy in head and neck cancers

- immunotherapy in India

- immunotherapy in recurrent head and neck cancers

- treatment of cisplatin refractory head and neck cancers

- treatment of recurrent head and neck cancers
Background Recurrent metastatic head and neck squamous cell carcinoma (HNSCC) patients carry a poor prognosis and have limited therapeutic options. In the randomized phase-3 trial CheckMate 141, nivolumab showed benefit in overall survival (OS) with manageable toxicity. Nivolumab is available for clinical practice since 2017 in India. The aim of this study is to evaluate the efficacy and safety of nivolumab in real-world settings in India.

Materials and Methods This is a retrospective, single-center study on the use of nivolumab with advanced or metastatic HNSCC in India. Eligible patients had histologically confirmed, recurrent squamous cell carcinoma of the head and neck (including metastatic disease) of the oral cavity, pharynx, or larynx that was not amenable to curative treatment, tumor progression, or recurrence after the administration of platinum-containing chemotherapy administered as adjuvant therapy or in the context of primary or recurrent disease. We assessed demographics, safety (the Common Terminology Criteria for Adverse Events Version 4.0), response evaluation (the Response Evaluation Criteria in Solid Tumors Version 1.1), progression-free survival (PFS), and OS.

Results Among patients with platinum-refractory, recurrent HNSCC, and treatment with nivolumab resulted in median PFS of 2 months and median OS of 5 months, which is inferior to what was seen in CheckMate 141 . Fifteen of 20 patients (75\%) had progressive disease, 3 (15\%) showed a partial response, and 2 (10\%) had stable disease.

Conclusion Nivolumab was well tolerated in our study with fewer toxic effects, and an inferior median survival was reached as compared with CheckMate 141 in platinum refractory, recurrent HNSCC patients treated with nivolumab because $90 \%$ of patients in our study received nivolumab as second-line therapy after progression. Our study encourages the use of nivolumab in this population.
DOI https://doi.org/10.1055/s-0041-1729443 ISSN 2278-330X

How to cite this article: Abbas W, Gupta S, Goel V, et al. RealWorld Experience of Immunotherapy from India in Recurrent Squamous Cell Carcinoma of Head and Neck Cancer. South Asian J Cancer 2021;10(2):72-75. (c) 2021. MedIntel Services Pvt Ltd.

MedIntel Services Pvt Ltd.. This is an open access article published by Thieme under the terms of the CreativeCommons Attribution-NonDerivative-NonCommercial-License, permitting copying and reproduction so long as the original work is given appropriate credit. Contents may not be used for commercial purposes, or adapted, remixed, transformed or built upon. (https://creativecommons.org/licenses/by-nc-nd/4.0/). Thieme Medical and Scientific Publishers Private Ltd A-12, Second Floor, Sector -2, NOIDA -201301, India 


\section{Introduction}

Squamous carcinoma of head and neck encompasses a diverse group of tumors with aggressive biological behavior. Head and neck cancer is the second most common cancer in India with 122,834 deaths and 205,321 cases diagnosed in 2018. ${ }^{1}$ Higher incidence of head and neck cancers is seen in India, compared with other malignancies which could be due to excessive consumption of tobacco in various forms and spectrum varying from place to place within the country, attributed to variation in regional risk factors. ${ }^{2}$ Most patients with head and neck squamous cell carcinoma (HNSCC) present with locoregionally advanced disease, and 30 to $40 \%$ of cases have a recurrence within 2 years. ${ }^{3-5}$ Survival benefit with the second-line treatment is modest, with response rates (RRs) of 5 to $10 \%$ and median progression-free survival (PFS) of 2 to 4 months. Thus, there was an unmet need for the second-line treatment options capable to improve efficacy. ${ }^{6}$ The 2016 Food and Drug Administration approval of the programmed death-1 (PD-1) monoclonal antibodies pembrolizumab and nivolumab for the treatment of HNSCC heralded the dawn of a new era of treatment for a patient population that historically has a 50\% RR despite aggressive multimodality treatment involving surgery, radiotherapy, chemotherapy, and epidermal growth factor receptor inhibition. ${ }^{7,8}$

The addition of targeted agent cetuximab, prolonged the median PFS time from 3.3 to 5.6 months and increased the response rate from 20 to $36 \%{ }^{9}$

Two checkpoint inhibitors, nivolumab ${ }^{10}$ and pembrolizumab, ${ }^{11}$ have been approved for patients with recurrent metastatic squamous cell carcinoma of the head and neck. KEYNOTE-040 by Cohen et al and CheckMate 141 by Ferris et al study showed significantly improved median overall survival (OS) and PFS with pembrolizumab and nivolumab, respectively, compared with the standard therapy. KEYNOTE-040 established PD-ligand 1 (PD-L1) combined positive score as a valid marker for head and neck cancer that should be routinely measured in these patients.

KEYNOTE-048 showed improved OS with the first-line pembrolizumab alone compared with the standard regimen of platinum, fluorouracil, and cetuximab. ${ }^{12}$

Experience in routine clinical practice may differ from that seen in a controlled clinical trial. This is a retrospective study that represents the real-world experience in previously treated head and neck cancers. The main aim of this study is to analyze the treatment outcomes and safety of patients treated with immunotherapy in the second-line setting, failing after platinum-based therapy.

\section{Materials and Methods}

In this retrospective, single-center study performed in a tertiary care hospital in India, 20 eligible patients were analyzed from June 2017 to March 2019. Eligibility criteria included histologically confirmed squamous cell head and neck cancer, prior therapy (including concurrent chemoradiotherapy, surgery, and platinum-based chemotherapy), minimum two doses of nivolumab, and the Eastern Cooperative Oncology Group (ECOG) performance status (PS) 0-1. Patients received nivolumab (at a dose of $3 \mathrm{mg} / \mathrm{kg}$ of body weight) at every 2 weeks until progressive disease or unacceptable toxicity. The main aim of the study was to report the efficacy and safety profile of nivolumab in squamous cell head and neck cancer patients who recurred on prior therapy in the real world.

The PFS was calculated from the starting date of nivolumab until relapse or death. The OS was calculated from the starting date of nivolumab until the last date of the follow-up or death of the patient. The primary endpoint was OS. Additional endpoints included PFS, objective RRs, and safety.

Tumor response was assessed using the Response Evaluation Criteria in Solid Tumors Version 1.1. Safety was assessed using the Common Terminology Criteria for Adverse Events Version 4.0. OS and PFS were computed by the KaplanMeier method, with the log-rank test.

Quality of life was not assessed in our study. IBM SPSS Statistics, Version 20.0.Armonk, New York. (Copyright IBM Corporation and its licensors 1989, 2011) was used for data collection and analysis. This study was approved by the ethics committee.

\section{Results}

Twenty eligible, squamous cell head and neck cancer patients of Asian race, who recurred on prior therapy and received a minimum of two doses of nivolumab, were retrospectively analyzed from June 2017 to March 2019 and selected for the study from a single center. The median duration of follow-up was 12 months. Prior therapy included concurrent chemoradiotherapy, ${ }^{8}$ surgery followed by radiation, ${ }^{12}$ or platinum-based chemotherapy. ${ }^{2}$ The dose of nivolumab received was $3 \mathrm{mg} / \mathrm{kg}$ of bodyweight intravenous over 60 minute every 2 weeks.

Demographics revealed 12 of the 20 selected patients were males, with age of $\leq 60$ years and positive smoking history. Eight patients were females and nonsmokers of age more than 61 years. Majority of patients, 16, had ECOG PS of 1 . The oral cavity was the site of the primary tumor in 16 of 20 selected patients, while the rest four patients had pharynx as the primary site ( - Table 1 ).

Demographics including smoking status (majority positive), sex (majority males), ECOG PS (majority 1), and site of the primary tumor (majority oral cavity) were similar to CheckMate $141 .^{10}$ PD-L1 and p16 tumor status were not reported for the patients included.

Nivolumab was administered as first-line palliative treatment in two patients (10\%), second line in 14 patients (70\%), and in third line or subsequent lines in four patients (20\%). The median number of doses administered was five. The objective response rates were $15 \%$ with three partial responses (-Table 2). No confirmed hyperprogression was observed. Median time since the last dose of platinum was 1 month. The median OS was 5 months (95\% confidence interval [CI]: 4.52-5.48), and the median PFS was 2 months (95\% CI: 1.38-2.62; -Figs. 1 and 2). 
Table 1 Patient demographics

\begin{tabular}{|c|c|c|}
\hline Patient characteristics & $\begin{array}{l}\text { Number of } \\
\text { patients ( } n)\end{array}$ & $\begin{array}{l}\text { Percentage of } \\
\text { patients (\%) }\end{array}$ \\
\hline \multicolumn{3}{|l|}{ Age (y) } \\
\hline$\leq 60$ & 12 & 60 \\
\hline$\geq 61$ & 8 & 40 \\
\hline \multicolumn{3}{|l|}{ Sex } \\
\hline Male & 12 & 60 \\
\hline Female & 8 & 40 \\
\hline \multicolumn{3}{|c|}{ History of smoking or tobacco } \\
\hline Yes & 12 & 60 \\
\hline No & 8 & 40 \\
\hline \multicolumn{3}{|l|}{$\mathrm{ECOG}^{\mathrm{a}}$} \\
\hline 0 & 0 & 0 \\
\hline 1 & 16 & 80 \\
\hline 2 & 4 & 20 \\
\hline \multicolumn{3}{|l|}{ Site of primary tumor } \\
\hline Larynx & 0 & 0 \\
\hline Oral cavity & 16 & 80 \\
\hline Pharynx & 4 & 20 \\
\hline \multicolumn{3}{|l|}{ Cetuximab received } \\
\hline Yes & 3 & 15 \\
\hline No & 17 & 85 \\
\hline \multicolumn{3}{|l|}{$\begin{array}{l}\text { Palliative nivolumab adminis- } \\
\text { tered as }\end{array}$} \\
\hline First line & 2 & 10 \\
\hline Second line & 14 & 70 \\
\hline Third line & 4 & 20 \\
\hline
\end{tabular}

Abbreviation: Eastern Cooperative Oncology Group.

aECOG performance status is a score ranging from 0 to 5 with 5 being dead and 0 being fully active.

Table 2 Response to nivolumab

\begin{tabular}{|l|l|l|}
\hline $\begin{array}{l}\text { Response to } \\
\text { nivolumab }\end{array}$ & $\begin{array}{l}\text { Number of } \\
\text { patients }(\boldsymbol{n})\end{array}$ & $\begin{array}{l}\text { Percentage of } \\
\text { patients (\%) }\end{array}$ \\
\hline Progressive disease & 15 & 75 \\
\hline Stable disease & 2 & 10 \\
\hline Partial response & 3 & 15 \\
\hline Complete response & 0 & 0 \\
\hline
\end{tabular}

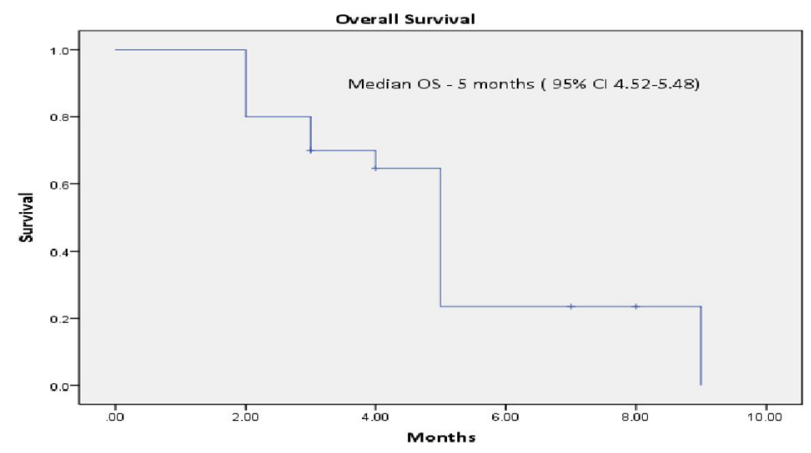

Fig. 1 Median overall survival (OS). Cl, confidence interval.

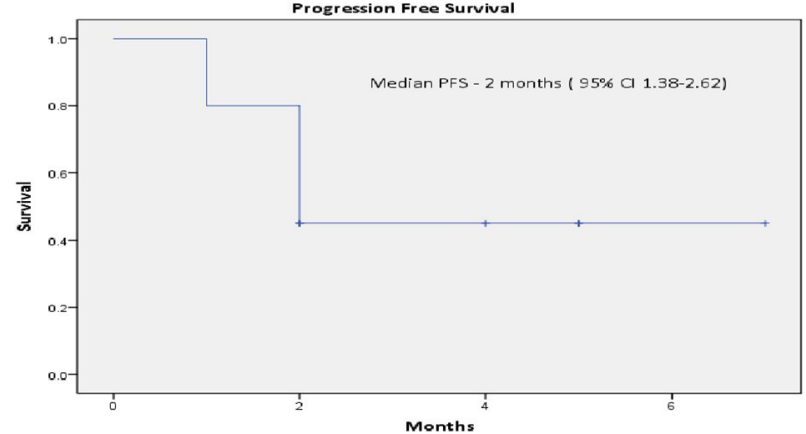

Fig. 2 Median progression-free survival (PFS). Cl, confidence interval.

Table 3 Side effects with nivolumab

\begin{tabular}{|l|l|l|}
\hline Side effects & Grades $\mathbf{1}$ and $\mathbf{2}$ & Grades $\mathbf{3}$ and 4 \\
\hline Fatigue & 4 & \\
\hline Nausea & 2 & \\
\hline Vomiting & 1 & \\
\hline Diarrhea & 1 & \\
\hline Stomatitis & 1 & \\
\hline Rash & 2 & \\
\hline Alopecia & 1 & \\
\hline Pneumonitis & & 2 \\
\hline
\end{tabular}

\section{Adverse Events}

The most frequent treatment-related adverse events were grade 1 or 2 . Fewer toxic grade 3 or 4 event $^{2}$ was observed. The most common event seen was fatigue. ${ }^{4}$ Majority of gastrointestinal events, such as nausea, ${ }^{2}$ vomiting, ${ }^{1}$ and diarrhea, ${ }^{1}$ were infrequent and of grade 1 or 2 . Side effects, such as stomatitis, ${ }^{1}$ rash, ${ }^{2}$ and alopecia, ${ }^{1}$ were also infrequent. No treatment-related deaths were seen in the study. Pneumonitis was seen in two patients who were managed with corticosteroids ( - Table 3 ).

\section{Discussion}

Nivolumab was well tolerated in our study with fewer toxic effects, and an inferior median survival was reached as compared with CheckMate 141 in platinum refractory, recurrent squamous cell carcinoma of head and neck patients treated with nivolumab. To the best of author's knowledge, this is the first of its kind study from India. Reason for inferior median PFS and OS achieved in our study compared with CheckMate 141 could be 44\% of nivolumab group patients in CheckMate 141 were started on nivolumab upfront after progression compared with majority of patients (90\%) in our study received nivolumab as the second line after progression. In CheckMate 141, nivolumab group patients with PD-L1 expression of more than $1 \%$ showed better median survival (8.8 months) compared with 5.7 months 
median survival in patients with PD-L1 expression of $<1 \%$. Nivolumab group patients with positive p16 status in CheckMate 141 showed a superior median survival of 9.1 months compared with 7.5 months in p16-negative patients. PD-L1 expression and p16 tumor status were not assessed for patients included in our study. Hypothetically, if patients in our study had PD-L1 expression of $<1 \%$ and negative p16 status, results of our study would be similar to CheckMate 141. In CheckMate 141, the patient subset did not receive the standard of care therapy. The most common treatment-related adverse events seen in our study (grades 1 and 2 fatigue, nausea, and rash) were consistent with those seen in CheckMate $141 .^{10}$

\section{Limitations}

Limitations of our study are as follows: first, it is a single centric, retrospective study with small sample size and lack of PD-L1 expression and p16 tumor status assessment; Second, given the impact of costs and tolerability, patient selection has to take place to provide individualized treatment, sparing toxicities, and maximizing clinical outcomes.

\section{Conclusion}

Treatment with nivolumab in platinum refractory, recurrent metastatic head and neck cancer patients, although resulted in an inferior median survival compared with CheckMate 141, was well tolerated in our study, with fewer grade- 3 adverse events and no treatment-related deaths. Further novel studies and therapies are required to address the unmet need for treatment of recurrent metastatic head and neck cancer. This clinically relevant data support the use of nivolumab in metastatic head and neck cancer who recurred on prior therapy.

\footnotetext{
Funding

The author(s) received no financial support for the research
}

Conflict of Interest

There are no conflicts of interest to declare.

\section{References}

1 Bray F, Ferlay J, Soerjomataram I, Siegel RL, Torre LA, Jemal A. Global cancer statistics 2018: GLOBOCAN estimates of incidence and mortality worldwide for 36 cancers in 185 countries. CA Cancer J Clin 2018;68(6):394-424

2 Franceschi S, Bidoli E, Herrero R, Muñoz N. Comparison of cancers of the oral cavity and pharynx worldwide: etiological clues. Oral Oncol 2000;36(1):106-115

3 Elango JK, Gangadharan P, Sumithra S, Kuriakose MA. Trends of head and neck cancers in urban and rural India. Asian Pac J Cancer Prev 2006;7(1):108-112

4 Bhattacharjee A, Chakraborty A, Purkaystha P. Prevalence of head and neck cancers in the north east-An institutional study. Indian J Otolaryngol Head Neck Surg 2006;58(1):15-19

5 Mehrotra R, Singh M, Gupta RK, Singh M, Kapoor AK. Trends of prevalence and pathological spectrum of head and neck cancers in North India. Indian J Cancer 2005;42(2):89-93

6 Wiegand S, Zimmermann A, Wilhelm T, Werner JA. Survival after distant metastasis in head and neck cancer. Anticancer Res 2015;35(10):5499-5502

7 Argiris A, Karamouzis MV, Raben D, Ferris RL. Head and neck cancer. Lancet 2008;371(9625):1695-1709

8 Steinbichler TB, Lichtenecker M, Anegg M, et al. Persistent head and neck cancer following first-line treatment. Cancers (Basel) 2018;10(11):10

9 Vermorken JB, Mesia R, Rivera F, et al. Platinum-based chemotherapy plus cetuximab in head and neck cancer. N Engl J Med 2008;359(11):1116-1127

10 Ferris RL, Blumenschein G Jr., Fayette J, et al. Nivolumab for recurrent squamous-cell carcinoma of the head and neck. N Engl J Med 2016;375(19):1856-1867

11 Cohen EE, Machiels J-PH, Harrington KJ, et al. KEYNOTE-040: a phase III randomized trial of pembrolizumab (MK-3475) versus standard treatment in patients with recurrent or metastatic head and neck cancer. J Clin Oncol 2017;33(15):156-167

12 Burtness B, Harrington KJ, Greil R, et al. KEYNOTE-048 Investigators. Pembrolizumab alone or with chemotherapy versus cetuximab with chemotherapy for recurrent or metastatic squamous cell carcinoma of the head and neck (KEYNOTE-048): a randomised, open-label, phase 3 study. Lancet 2019;394(10212):1915-1928 